\title{
Outbreak
}

\section{Molecular and epidemiologyical analysis of a Campylobacter jejuni outbreak in China, 2018}

\author{
Mei Qu ${ }^{1}$, Maojun Zhang ${ }^{2}$, Xin Zhang ${ }^{1}$, Lei Jia ${ }^{1}$, Jun Xư ${ }^{3}$, Yanhui Chu ${ }^{3}$ Zhichao Liang ${ }^{1}$, Bing Lv ${ }^{1}$, Hao \\ Liang $^{2}$, Ying Huang ${ }^{1}$, Quanyi Wang ${ }^{1}$ \\ ${ }^{1}$ Beijing Key Laboratory of Diagnostic and Traceability Technologies for Food Poisoning, Institute of Infectious and \\ Endemic Diseases Control, Beijing Center for Disease Prevention and Control, Beijing Research Center for \\ Preventive Medicine, Beijing, 100013, China \\ ${ }^{2}$ State Key Laboratory for Infectious Disease Prevention and Control, National Institute for Communicable Disease \\ Control and Prevention, Changping, Beijing, 102206, China \\ ${ }^{3}$ Beijing Xicheng District Center for Diseases Control and Prevention, China
}

\begin{abstract}
Introduction: Campylobacter spp. is the most common gastrointestinal pathogen worldwide with a very low reported incidence in China. In April 2018, one 36 cases of diarrhea outbreak occurred in a high school in Beijing after a trip to another province in Southern China. The investigation for the enteric pathogen infection was conducted to identify the cause.

Methodology: Eighteen stool specimens from 11 diarrheal patients and 3 close contacts were collected and tested for 16 enteric bacterial and viral pathogens using real-time PCR methods. Multilocus Sequence Typing (MLST), pulsed-field gel electrophoresis (PFGE) and antimicrobial susceptibility testing were applied to $C$. jejuni isolates in this outbreak in order to characterize the incident.

Results: Ten (90.9\%) of 11 stool specimens from diarrheal patients were positive for Campylobacter jejuni, confirmed as the cause of outbreak. Eight $C$. jejuni strains were obtained and produced 2 sequence types (STs) and 3 PFGE patterns. Six of them had the same ST (ST2274) and PFGE pattern (SMA001). The dominant outbreak strain with an identical subtyping profile was clustered with most chicken isolates from national MLST and PulseNet Campylobacter database. The entire 8 isolates were multi-drug resistant, with the dominant resistance pattern of nalidixic acid, tetracycline and ciprofloxacin combined, except for 2 isolates resistant to florfenicol.

Conclusion: Molecular typing confirmed that most of the cases belonged to a clonal cluster supporting the hypothesis of a common source; however, the source was not identified. This was the first recognized Campylobacteriosis outbreak in China among 20 years.
\end{abstract}

Key words: outbreak; Campylobacter jejuni; diarrhea; molecular subtyping.

J Infect Dev Ctries 2019; 13(12):1086-1094. doi:10.3855/jidc.11408

(Received 28 February 2019 - Accepted 10 June 2019)

Copyright (C) 2019 Qu et al. This is an open-access article distributed under the Creative Commons Attribution License, which permits unrestricted use, distribution, and reproduction in any medium, provided the original work is properly cited.

\section{Introduction}

Campylobacter spp., as foodborne zoonotic pathogen, is recognized to be the leading cause of human bacterial gastroenteritis both in developed and developing countries [1-3]. Campylobacter is transmitted to humans through the consumption of raw milk, water, or contaminated meat, particularly in poultry [4]. It was estimated that in 2010, Campylobacter spp. caused the highest number of laboratory-confirmed food-borne bacterial infections worldwide (96 million, 95\% UI: 51-177) [5]. In the United States annually, Campylobacteriosis has been estimated to affect 7 million people annually, causing $110-511$ deaths and costing \$1.2-\$6 billion [6, 7]. In the European Union, the incidence of human Campylobacteriosis cases has followed an increasing trend in recent times and it continues to be the most commonly reported zoonosis with 214268 confirmed cases in 2012 [8]. The majority of cases are sporadic and outbreaks of Campylobacter infection are rarely reported [9].

In China, Campylobacter infections are not subject to mandatory notification and Campylobacter surveillance is on a voluntary basis by local and regional laboratories. In part due to insensitive conventional isolation methods, there were not many cases of Campylobacter infection detected by monitoring since 2000 [10, 11]. Although recently, a modified membrane filtration culture, has led to increased identification of a broad range of Campylobacter [12], there have been no outbreak reports so far.

The aim of this study was to describe both the epidemiological and microbiological investigation of $C$. 
jejuni outbreak that occurred in high school in Beijing after a trip to southern province in China during April 2018 .

\section{Methodology}

Epidemiological investigation

From April 8 to 16, 36 cases of acute gastroenteritis were reported from the Xicheng $\mathrm{CDC}$, a local district $\mathrm{CDC}$ in Beijing. All of the cases were students and teachers from a high school in Beijing. The local public health authorities conducted the investigation for enteric pathogen infection to identify the cause. According to the investigation, from April 7 to 11, one trip from Beijing to Jingdezhen City in Jiangxi Province in Southern China, was organized by the school. The first patient developed symptoms at approximately 20:00. on April 8 when they were in Jingdezhen City. From April 8 to 16, a total of 36 persons were affected and had common exposure to food and environment during this trip. Of these 36 patients with the main symptoms of diarrhea, accompanying with fever, vomiting and abdominal pain, 26 cases went to the hospital for treatment, and 21 cases were diagnosed as acute infectious diarrhea. Till April 20, a total of 33 cases had recovered. A probable case was defined as a school member with diarrhea onset within 5 days on or after taking a trip April 7-11. Because the microbiological results later indicated that the outbreak was caused by C. jejuni, the definition for confirmed cases was chosen accordingly as: those who had a laboratory-confirmed diagnosis of C. jejuni.

\section{Sample collection}

As a part of the investigation, the stool samples were collected for the routine detection of 11 bacteria and 5 viruses in the microbiological laboratory in Beijing CDC. In total, 18 stool samples including 14 frozen and 4 fresh stool samples, were collected during the period of April 14 to May 8 (Table 1). In initial stage of this outbreak, 5 stool samples from 5 diarrheal patients were collected for preliminary etiological identification on April 14 to 17. The Campylobacter jejuni, adenovirus and rotavirus were found by realtime PCR detection of 16 enteric pathogens. Since 4 of 5 cases were confirmed as $C$. jejuni infection consistent with clinical symptoms, the subsequent 9 samples including 6 from the patients and 3 from the close contacts were screened for $C$. jejuni on April 18. These 14 feces specimens were frozen and preserved at $-20^{\circ} \mathrm{C}$ before being tested. During the recovery period, 4 fresh stool samples from the 4 patients mentioned above were collected for the re-examination of Campylobacter on May 8.

\section{DNA extraction and real-time PCR detection}

The viral and bacterial nucleic acid was extracted and purified from the stool specimen using QIAamp Viral RNA Mini Kit (Qiagen, Hilden, Germany) and QIAamp DNA Stool Mini Kit (Qiagen, Hilden, Germany), respectively, according to the manufacturer's recommended protocols. Simultaneously, the initial analyses were performed by real-time PCR assays for Salmonella, Shigella, diarrheagenic Escherichia coli, Vibrio

Table 1. Sample information and detection results in the diarrhea outbreak caused by C. jejuni, China, April to May 2018.

\begin{tabular}{|c|c|c|c|c|c|c|c|c|c|}
\hline \multirow{2}{*}{$\begin{array}{c}\text { Sample } \\
\text { ID }\end{array}$} & \multirow{2}{*}{ Source } & \multirow{2}{*}{ Occupation } & \multirow{2}{*}{ Onset time } & \multirow{2}{*}{$\begin{array}{c}\text { Sampling } \\
\text { time }\end{array}$} & \multirow{2}{*}{$\begin{array}{l}\text { Detection } \\
\text { time }\end{array}$} & \multirow{2}{*}{$\begin{array}{l}\text { Specimen } \\
\text { status }\end{array}$} & \multicolumn{2}{|c|}{ Real-time PCR } & \multirow{2}{*}{$\begin{array}{c}\text { Isolation } \\
\text { of }\end{array}$} \\
\hline & & & & & & & Pathogen & Ct-value* & \\
\hline 1 & patient & student & 2018-4-12 & $2018-4-14$ & $2018-4-17$ & frozen stool & C. jejuni & 34.0 & negative \\
\hline 2 & patient & student & $2018-4-13$ & $2018-4-16$ & $2018-4-17$ & frozen stool & C. jejuni & 16.2 & positive \\
\hline 3 & patient & student & $2018-4-8$ & $2018-4-16$ & $2018-4-17$ & frozen stool & adenovirus & 31.6 & negative \\
\hline 4 & patient & student & 2018-4-12 & $2018-4-16$ & $2018-4-17$ & frozen stool & C. jejuni & 15.1 & positive \\
\hline 5 & patient & student & $2018-4-13$ & $2018-4-17$ & $2018-4-17$ & frozen stool & C. jejuni, rotavirus & $31.4,32.2$ & negative \\
\hline 6 & patient & teacher & $2018-4-13$ & $2018-4-18$ & $2018-4-27$ & frozen stool & C. jejuni & 20.3 & positive \\
\hline 7 & patient & student & 2018-4-12 & $2018-4-18$ & $2018-4-27$ & frozen stool & C. jejuni & 28.0 & positive \\
\hline 8 & patient & student & $2018-4-12$ & $2018-4-18$ & $2018-4-27$ & frozen stool & C. jejuni & 26.0 & positive \\
\hline 9 & patient & student & $2018-4-13$ & 2018-4-18 & $2018-4-27$ & frozen stool & C. jejuni & 18.7 & positive \\
\hline 10 & patient & teacher & 2018-4-12 & $2018-4-18$ & $2018-4-27$ & frozen stool & C. jejuni & 19.5 & positive \\
\hline 11 & close contact & student & - & $2018-4-18$ & $2018-4-27$ & frozen stool & negative & - & negative \\
\hline 12 & close contact & student & - & $2018-4-18$ & $2018-4-27$ & frozen stool & negative & - & negative \\
\hline 13 & patient & teacher & 2018-4-13 & $2018-4-18$ & $2018-4-27$ & frozen stool & C. jejuni & 32.4 & negative \\
\hline 14 & close contact & student & - & $2018-4-18$ & $2018-4-27$ & frozen stool & C. jejuni & 31.3 & negative \\
\hline $5-2$ & patient & student & as above 5 & $2018-5-8$ & $2018-5-8$ & fresh stool & C. jejuni & 19.7 & positive \\
\hline $13-2$ & patient & student & as above 13 & $2018-5-8$ & $2018-5-8$ & fresh stool & negative & - & negative \\
\hline $6-2$ & patient & student & as above 6 & $2018-5-8$ & $2018-5-8$ & fresh stool & negative & - & negative \\
\hline $10-2$ & patient & student & as above 10 & $2018-5-8$ & $2018-5-8$ & fresh stool & negative & - & negative \\
\hline
\end{tabular}


parahaemolyticus, Vibrio cholera, Listeria monocytogenes, Staphylococcus aureus, Campylobacter, Yersinia enterocolitica, Bacillus cereus, Clostridium perfringens, rotavirus, norovirus, adenovirus, astrovirus, sapovirus using kits (Bioperfectus technology Co., Ltd., Taizhou, Jiangsu Province, , China) in accordance with the manufacturer's instruction.

\section{Isolation and identification of Campylobacter}

In this study, Campylobacter spp. isolation was executed using a novel laboratory modified Campylobacter isolation kit incorporating a membrane filter method (ZC-CAMPY-002, Qingdao Sinova Biotechnology Co., Ltd., Qingdao, China) as described previously [12]. Briefly, $1 \mathrm{~mL}$ stool specimen suspension was transferred into $4 \mathrm{~mL}$ enrichment buffer which was provided in the kit. The principle component of the enrichment buffer was the modified Preston broth which was described in the manual book. The enriched suspension was incubated at $37^{\circ} \mathrm{C}$ for 24 hours under microaerophilic conditions consisting of $5 \% \mathrm{O}_{2}, 10 \%$ $\mathrm{CO}_{2}$, and $85 \% \quad \mathrm{~N}_{2}$. Three hundred $\mu \mathrm{L}$ cultured enrichment suspension was spotted on to the surface of the filter pasted on to the double medium plates, which contained Karmali and Columbia agar, respectively. The medium plates were incubated at $37^{\circ} \mathrm{C}$ for 48 hours under microaerophilic conditions. Further identification was performed with real-time PCR assay using primer sets specific to hipO gene in C. jejuni. Briefly, hipO gene TaqMan Probe: FAMATCCGAAGAAGCCATCATCGCACCT-TAMRA; forward primer: 5'-CCCCTTCCAATAACTTCAATG3'; reverse primer: 5'TGGCACAATATGCCTTTTGGTA-3. Template DNA for PCR was prepared by boiling method. At least five colonies of pure culture grown on Columbia blood agar plates was suspended in $100 \mu \mathrm{L}$ of sterile water. The cell suspension was boiled at $100^{\circ} \mathrm{C}$ for 10 minutes and centrifuged at $12000 \mathrm{rpm}$ for 5 minutes, $2 \mu \mathrm{L}$ of the supernatant was used as a template for the real-time PCR. The real-time PCR assay was performed using the TaqMan Universal PCR Master Mix Kit (Applied Biosystems, Edison, United States) on Applied Biosystems 7500 Real-Time PCR System. The amplification profile consisted of heat activation at $95^{\circ} \mathrm{C}$ for 10 minutes; 40 cycles of denaturation at $95^{\circ} \mathrm{C}$ for $15 \mathrm{~s}$, annealing, extension, and fluorogenic signal detection at $60^{\circ} \mathrm{C}$ for 1 minute. The assay was considered positive when the number of cycles to detection was 35 or less with the standard S curve.
Multilocus sequence typing (MLST) and Pulsed-field gel electrophoresis (PFGE)

MLST was performed by amplifying and sequencing seven housekeeping gene loci (asp A, gln $\mathrm{A}$, glt $\mathrm{A}, g l y \mathrm{~A}, p g m$, tkt, and $u n c \mathrm{~A})$ using previously described primers [13]. The sequence data for each of the seven MLST loci were compared with sequences in the MLST database (www.pubmlst.org/campylobacter) to determine the allele number. The sequence type (ST) of each strain was assigned from the profiles of the seven alleles in the MLST database.

PFGE was performed according to the standard PulseNet PFGE protocol for C. jejuni (http://www.cdc.gov/pulsenet/protocols/

campy_protocol.pdf). All the isolates in this study were analyzed by digestion with $S m a$ I. PFGE cluster analysis was performed using BioNumerics (Version 5.1; Applied Maths) and the Dice coefficient with a $1.5 \%$ tolerance window was determined using the unweight pair group method with arithmetic averages (UPGMA). The strains were verified for outbreak isolates on the basis of their PFGE profile similarity and having the same MLST.

\section{Antimicrobial susceptibility testing}

The Minimum Inhibitory Concentrations (MICs) for 11 antimicrobials on Campylobacter antimicrobial resistance monitoring panel from Chinese Pathogen Identification Net, were determined using an agar dilution method as recommended by CLSI (M100-S25, 2015). Antimicrobial susceptibility was evaluated using the quality control strain C. jejuni ATCC 33560. Briefly, suspensions of $C$. jejuni isolates adjusted to 0.5 McFarland (approximately $10^{8} \mathrm{CFU} / \mathrm{mL}$ ) were prepared in phosphate buffered saline and inoculated onto Mueller-Hinton agar (CM0337, Oxoid, Basingstoke, United Kingdom) plates supplemented with 5\% defibrinated sheep blood and antimicrobial agents in concentrations ranging from 0.25 to $64 \mu \mathrm{g} / \mathrm{mL}$ and incubated under microaerophilic conditions at $37^{\circ} \mathrm{C}$ for $48 \mathrm{~h}$. The MIC interpretive criterion for resistance in this study according to the standard used in the National Antimicrobial Resistance Monitoring System (NARMS-2014) for Campylobacter in the United States: erythromycin $(\geq 32 \mu \mathrm{g} / \mathrm{mL})$, azithromycin $(\geq$ $8 \mu \mathrm{g} / \mathrm{mL})$, nalidixic acid $(\geq 6 \mu \mathrm{g} / \mathrm{mL})$, ciprofloxacin $(\geq$ $4 \mu \mathrm{g} / \mathrm{mL})$, gentamicin $(\geq 8 \mu \mathrm{g} / \mathrm{mL})$, streptomycin $(\geq$ $16 \mu \mathrm{g} / \mathrm{mL})$, chloramphenicol $(\geq 32 \mu \mathrm{g} / \mathrm{mL})$, florfenicol $(8 \mu \mathrm{g} / \mathrm{mL})$, tetracycline $(\geq 16 \mu \mathrm{g} / \mathrm{mL})$, telithromycin $(\geq$ $16 \mu \mathrm{g} / \mathrm{mL})$, and clindamycin $(\geq 8 \mu \mathrm{g} / \mathrm{mL})$. 


\section{Results}

Epidemiological findings

On April 7 to 11, one trip from Beijing to Jingdezhen City was organized by the school. Of the 161 school members (138 students, 23 teachers) involved in the trip, 36 had acute gastroenteritis (attack rate (AR): $22.4 \%$ ). Of these 36 people (17 males, 19 females), 32 (88.9\%) were students, and 4 (11.1\%) were teachers. The age of 32 students was 15 or 16 years old and the age of 4 teachers ranged from 27 to 40 years old. The main symptoms were diarrhea (97.2\%). Abdominal pain (63.9\%), fever (55.6\%), nausea $(22.2 \%)$ and vomiting $(13.9 \%)$ were less frequent. Day of illness onset for the 36 cases is shown in Figure 1. The first patient developed symptoms on April 8, however, who was not confirmed by laboratory $C$. jejuni infection analysis. After a slight increase beginning on April 11, the number of cases rose sharply to a distinct peak on April 12 and 13, and decreased exponentially in the following day, and then disappeared completely. The epidemic curve showed characteristics of a point source outbreak [14]. The date of exposure was estimated to be April 10 and 11, considering the peak of symptom development on April 12 to 13 , and an average incubation period of 1-5 days for enteric pathogen infection. As food and environmental specimens were not available, the menu logbook was reviewed to identify a possible source of the outbreak. The suspicious food mainly included stewed chicken, duck, fried chicken and braised lamb for dinner on April 10, and beef, shrimp, sauce duck, lamb and chicken meat for lunch on April 11.

\section{Laboratory findings}

A total of 18 stool samples comprise 14 cases obtained from 11 symptomatic people and 3 close contacts onset, and 4 cases re-collected samples in the recovery period (Table 1 ). Of the 18 stool samples, 11 were positive for $C$. jejuni, 1 for adenovirus, and 1 for C. jejuni and rotavirus co-infection, the other 5 for negative results, using real-time PCR assay. The only two cases of virus infection effectively excluded virus as the causative pathogen of this outbreak.

The filtration culture method was used for the $C$. jejuni isolation in this investigation. Twelve stools samples with laboratory-confirmed Campylobacter by PCR were frozen before Campylobacter culture. Among these frozen stools, a total of 8 C. jejuni strains were isolated, which all were provided from patients. The other 4 stool samples, despite positive for nucleic acid detection, proved Campylobacter culture negative due to low infection amount $(\mathrm{Ct}>31)$.
Figure 1. Cases of diarrhea illness in traveler's outbreak by date of onset, April 8-16, $2018(\mathrm{n}=36)$.

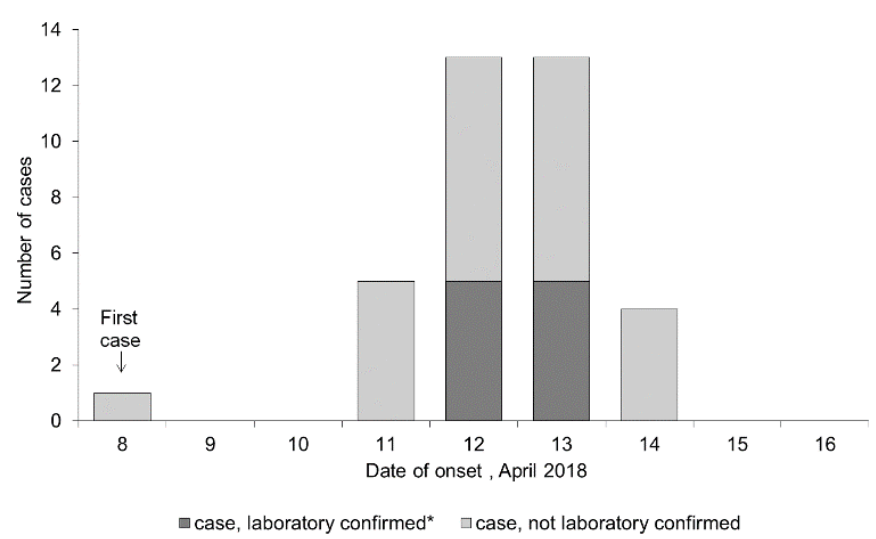

The first case on April 8 was confirmed by adenovirus infection rather than C. Jejuni. * The case, laboratory confirmed was verified by $C$. Jejuni.

\section{Molecular subtyping and antibiotic susceptibility}

MLST and PFGE were applied to 8 C. jejuni isolates in this outbreak in order to characterize the incident. Two STs 2274 and 9224 were identified from 8 isolates by MLST. Among them, 7 strains (CJ002, CJ004, CJ005, CJ006, CJ007, CJ008 and CJ009) were assigned to ST2274, which was more common and widely distribute, not belonged to any known clonal complex. When compared with those of 41 available sporadic clinical strains and 7 chicken isolates from national MLST database, ST2274 was clustered with chicken isolates (Figure 2). ST9224 from strain CJ010 is a newly discovered sequence type with the new allele 922 at pgm gene locus.

By PFGE, 6 of 8 isolates showed indistinguishable macrorestriction patterns (SMA001), and the other 2 isolates (CJ009 and CJ010) yielded two different patterns (SMA002 and SMA003) respectively (Figure $3)$. When compared with those of the strains from national PulseNet database, the same type SMA001 was consistent with at least 7 chicken isolates. There was a strict correlation between PFGE and MLST genotypes for most isolates. Only one isolate CJ009's PFGE pattern SMA002 was distinguishable from the other 6 strains, while the ST remained identical. The strain CJ010's PFGE pattern SMA003, as a novel subtype, had not been recorded previously in the national PulseNet database.

All 8 C. jejuni isolates were resistant to nalidixic acid, tetracycline and ciprofloxacin in 11 tested antimicrobials. In addition, all strains were sensitive to erythromycin, azithromycin, gentamicin, streptomycin, 
chloramphenicol, telithromycin and clindamycin, except for that strains CJ009 and CJ010 were resistant to florfenicol (Table 2). Additional characterization of isolates by antimicrobial susceptibility testing identified the dominant outbreak strain as susceptible to 8 antimicrobials, but resistant to nalidixic acid, tetracycline and ciprofloxacin.

\section{Discussion}

From April 8 to 16 , a total of 36 people were affected and had common food exposure during this trip. According to the distribution of time and the number of onset people with rapid rise and decline in a short period of time, a single peak curve showed that it was a traveler's diarrhea outbreak. It is considered the possibility of foodborne contamination, although it is very difficult to trace the sources in this tour. In this outbreak, we conducted the isolation of Campylobacter from the frozen stool sample using the filtration method, which was evaluated as a more efficient means, consistent with other studies $[12,15,16]$.

Figure 2. Phylogenetic relatedness of 55 C. jejuni isolates. These strains included 8 isolates (CJ002, CJ004, CJ005, CJ006, CJ007, CJ008, CJ009 and CJ010) from this outbreak study, 41 isolates from sporadic clinical cases and 7 isolates from chicken fecal (BJ-CJH1, BJCJH11, BJ-CJH12, BJ-CJH17, BJ-CJH19, BJ-CJH20 and BJ-CJH24) from national MLST Campylobacter database. The minimum spanning tree showing the relatedness of 55 C. jejuni strains, which was based on the STs using Bionumerics software, version 5.1. Different circles correspond to different STs. The color of the circles indicates the number of isolates belonging to each ST. The 14 strains (CJ002, CJ004, CJ005, CJ006, CJ007, CJ008, CJ009, BJ-CJH1, BJ-CJH11, BJ-CJH12, BJ-CJH17, BJ-CJH19, BJ-CJH20 and BJ-CJH24) belonged to ST2274. However, the strain CJ010 from this outbreak produced a new sequence type 9924 with a new allele 922 at $p g m$ gene locus.

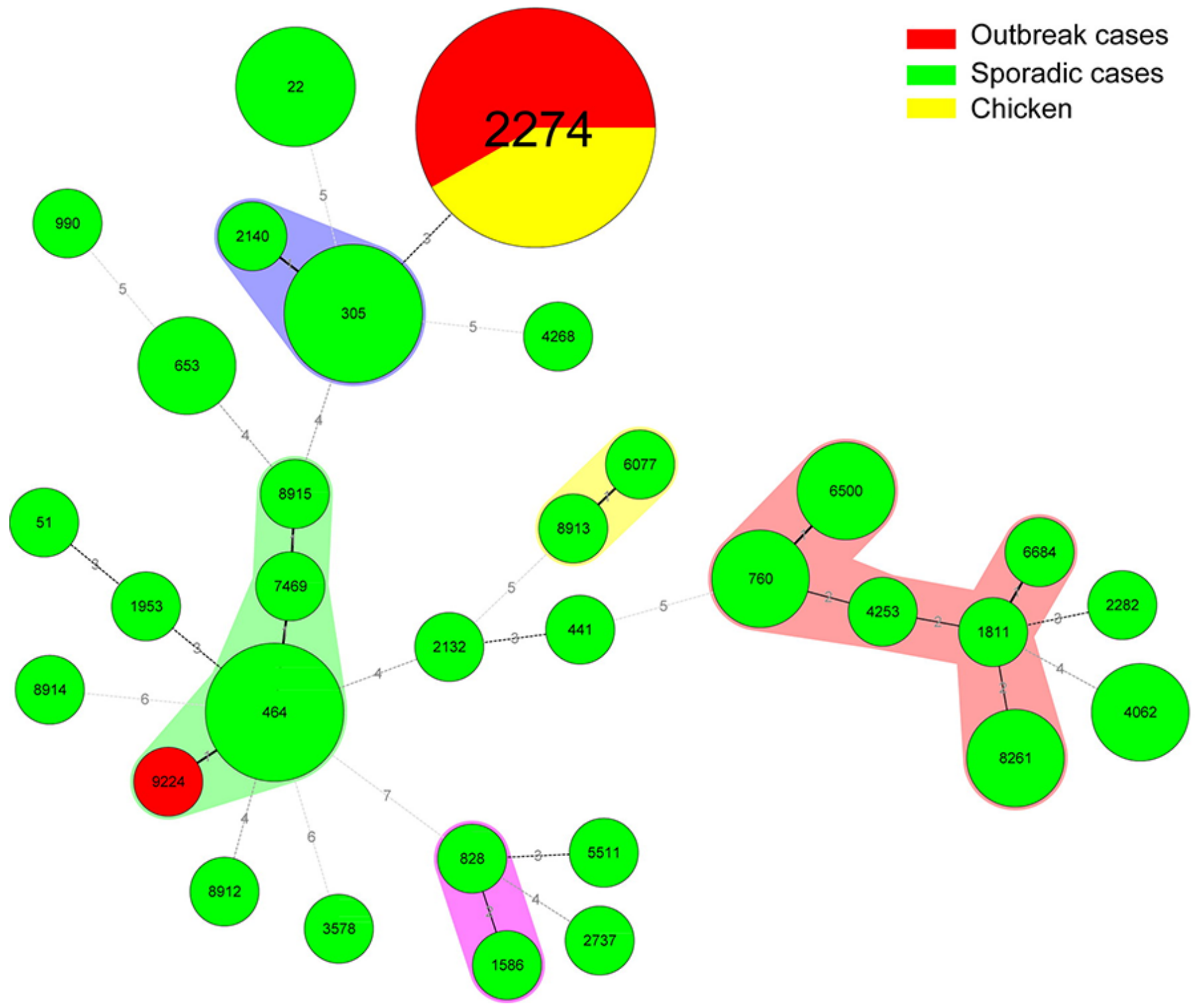


Table 2. Antimicrobial susceptibilities of 8 C. jejuni strains from patients in the diarrhea outbreak in China.

\begin{tabular}{lcccccccc}
\hline \multirow{2}{*}{ Antibiotic } & \multicolumn{1}{c}{ Susceptibility of strain } & & \\
\cline { 2 - 8 } & CJ002 & CJ004 & CJ005 & CJ006 & CJ007 & CJ008 & CJ009 & CJ010 \\
\hline Erythromycin & $\mathrm{S}$ & $\mathrm{S}$ & $\mathrm{S}$ & $\mathrm{S}$ & $\mathrm{S}$ & $\mathrm{S}$ & $\mathrm{S}$ & $\mathrm{S}$ \\
Azithromycin & $\mathrm{S}$ & $\mathrm{S}$ & $\mathrm{S}$ & $\mathrm{S}$ & $\mathrm{S}$ & $\mathrm{S}$ & $\mathrm{S}$ & $\mathrm{S}$ \\
Nalidixic acid & $\mathrm{R}$ & $\mathrm{R}$ & $\mathrm{R}$ & $\mathrm{R}$ & $\mathrm{R}$ & $\mathrm{R}$ & $\mathrm{R}$ & $\mathrm{R}$ \\
Ciprofloxacin & $\mathrm{R}$ & $\mathrm{R}$ & $\mathrm{R}$ & $\mathrm{R}$ & $\mathrm{R}$ & $\mathrm{R}$ & $\mathrm{R}$ & $\mathrm{R}$ \\
Gentamicin & $\mathrm{S}$ & $\mathrm{S}$ & $\mathrm{S}$ & $\mathrm{S}$ & $\mathrm{S}$ & $\mathrm{S}$ & $\mathrm{S}$ & $\mathrm{S}$ \\
Streptomycin & $\mathrm{S}$ & $\mathrm{S}$ & $\mathrm{S}$ & $\mathrm{S}$ & $\mathrm{S}$ & $\mathrm{S}$ & $\mathrm{S}$ & $\mathrm{S}$ \\
Chloramphenicol & $\mathrm{S}$ & $\mathrm{S}$ & $\mathrm{S}$ & $\mathrm{S}$ & $\mathrm{S}$ & $\mathrm{S}$ & $\mathrm{S}$ & $\mathrm{S}$ \\
Florfenicol & $\mathrm{S}$ & $\mathrm{S}$ & $\mathrm{S}$ & $\mathrm{S}$ & $\mathrm{S}$ & $\mathrm{S}$ & $\mathrm{R}$ & $\mathrm{R}$ \\
Tetracycline & $\mathrm{R}$ & $\mathrm{R}$ & $\mathrm{R}$ & $\mathrm{R}$ & $\mathrm{R}$ & $\mathrm{R}$ & $\mathrm{R}$ & $\mathrm{R}$ \\
Telithromycin & $\mathrm{S}$ & $\mathrm{S}$ & $\mathrm{S}$ & $\mathrm{S}$ & $\mathrm{S}$ & $\mathrm{S}$ & $\mathrm{S}$ & $\mathrm{S}$ \\
Clindamycin & $\mathrm{S}$ & $\mathrm{S}$ & $\mathrm{S}$ & $\mathrm{S}$ & $\mathrm{S}$ & $\mathrm{S}$ & $\mathrm{S}$ & $\mathrm{S}$ \\
\hline
\end{tabular}

Antibiotic resistance of $C$. jejuni is an increasing concern [17]. Fluoroquinolones are usually administered to treat human Campylobacteriosis in China [18]. Our results showed that all the tested isolates were resistant to nalidixic acid, ciprofloxacin and tetracycline. The three antibiotics were the most commonly reported resistance pattern consistent with those of previous studies [12, 19-21]. In Shanghai (Eastern China), more than $96 \%$ of the Campylobacter spp. isolates are resistant to quinolones and tetracycline [22]. In central China, even higher resistance of $C$. jejuni isolates from retail chicken meat has been reported: $100 \%$ resistance to ciprofloxacin and $90 \%$ resistanceto tetracycline [23]. Hence, a high prevalence of multidrug-resistant $C$. jejuni, which may be a threat to public safety in China, should be given great attention. We also found that two isolates were resistant to florfenicol, which is only licensed for use in animals. Nevertheless unexpected and rare, the florfenicolresistant Campylobacter was also observed in recent reports on the surveillance of diarrhea outpatient in Beijing and Shenzhen, two major cities in China [12, 24], which requires further investigation for the source and vehicles of such Campylobacter infection and to consider the selective pressure of antibiotics on $C$. jejuni strains in greater detail. It is noteworthy that there

Figure 3. PFGE Sma I dendrogram of 15 C. jejuni isolates. These strains included 8 isolates (CJ002, CJ004, CJ005, CJ006, CJ007, CJ008, CJ009 and CJ010) from this outbreak study and 7 isolates (BJ-CJH1, BJ-CJH11, BJ-CJH12, BJ-CJH17, BJ-CJH19, BJ-CJH20 and BJCJH24) from national PulseNet Campylobacter database. The levels of similarity were calculated using the Dice coefficient (1.5\% optimization and $1.5 \%$ position tolerance) and UPGMA for the cluster analysis. Three PFGE types (SMA001, SMA002 and SMA003) were identified. The PFGE type, MLST type and source are shown on the right.

Dice (Opt:1.50\%) (Tol 1.5\%-1.5\%) (H>0.0\% S>0.0\%) [0.0\%-100.0\%]
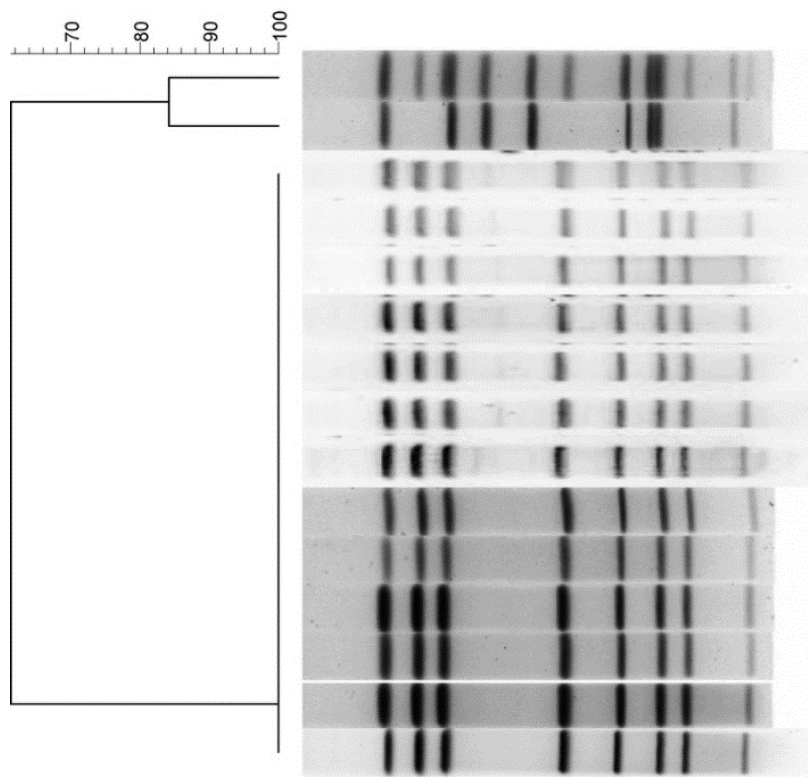

Isolates ID
CJ009
CJ010
BJ-CJH1
BJ-CJH11
BJ-CJH12
BJ-CJH17
BJ-CJH19
BJ-CJH20
BJ-CJH24
CJ002
CJ004
CJ005
CJ006
CJ007
CJ008

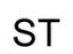

Human stool

Human stool

Chicken fecal

Chicken fecal

Chicken fecal

Chicken fecal

Chicken fecal

Chicken fecal

Chicken fecal

Human stool

Human stool

Human stool

Human stool

Human stool

Human stool
ST

2274

9224

2274

2274

2274

2274

2274

2274

2274

2274

2274

2274

2274

2274

2274
PFGE Smal pattern

SMA002

SMA003

SMA001

SMA001

SMA001

SMA001

SMA001

SMA001

SMA001

SMA001

SMA001

SMA001

SMA001

SMA001

SMA001 
are 6 isolates showed the same resistance pattern, which corroborates the finding of PFGE pattern and MLST subtyping. In addition, the outbreak strain was still available from one recovered patient, after 26 days of onset. It is implicated that the potential risk for $C$. jejuni could persist for a long time in an invisible infection manner and it is necessary to reinforce active surveillance.

Poultry is considered as the major reservoir for Campylobacter infection. Chicken meat is commonly considered to be the suspected food source for Campylobacter infection in humans $[25,26]$. In this study, we described epidemiological evidence for a human's diarrhea outbreak caused by $C$. jejuni and compared sequence types of isolates with animal isolate data sets. ST2274 is widely distributed throughout the world and closely related to infections in humans and chickens. As early as 2000, ST2274 was reported and prevalent among the sporadic gastroenteritis cases in Thailand. Then, from 2005 to 2011, it was more frequent in human, chicken, and chicken offal or meat in the United Kingdom, Belgium, Germany, Luxembourg and so on (from Campylobacter PubMLST database). In China, ST2274 was also common from sporadic diarrheal patient and chicken. Based on our investigation, chicken was also mentioned as consumed food in dinner on April 10 and in lunch on April 11. Furthermore, population genetic analysis indicated that the dominant outbreak strain with ST2274 and PFGE profile SMA001 was typical of isolates from chicken. Therefore, the source of $C$. jejuni infection in this outbreak was highly suspected to be chicken. The national surveillance data from 20 provinces in China demonstrated that the prevalence and risk assessment of $C$. jejuni in Chicken was relatively high $[27,28]$. The prevalence of $C$. jejuni in chicken meat was reported as $13.7 \%(31 / 227)$ in Tianjin [29] and $17.2 \%(52 / 302)$ in central China [23]. Handling and consumption of chicken meat are the major risk factors for human Campylobacteriosis [30]. It suggested that key efforts of food safety management strategies should be made to control the risk, especially in chick breeding and chicken preparation processes.

PFGE and MLST were useful to assess the strains in a clonal cluster isolated from the outbreak cases [31]. In this study, PFGE was able to discriminate strains within the same MLST. The predominant ST2274 was corroborated by the dominant PFGE pattern SAM001. While close examination of PFGE patterns SAM001 and SAM002 indicated an underlying similarity, with changes that suggested recent evolutionary divergence from a common ancestor type. Although one isolate had a slightly deviant PFGE pattern, this was not considered significant enough to exclude it from this outbreak, as a PFGE profile can change after only a single passage through the chicken host by genomic rearrangement [32]. Furthermore, since alterations in the PFGE patterns can result from a single-nucleotide polymorphism in a restriction site [33], a clonal relationship may exist even between strains with different PFGE profiles. Such changes may occur at relatively high frequency by the discriminatory power of PFGE compared with MLST and do not preclude our conclusion regarding the source of infection [34], since genotyping results must always be in context with the other results from the outbreak investigation.

One limitation in this report was that it was impossible to trace the potential origin of infection, because the food, water and environmental specimens were unavailable at the time of this survey. A second, the investigation did not begin until 2 days after the outbreak due to the notification delay, which resulted in a prolonged lag of sample collection and a limited amount of information. But this is the first report on a tourist's diarrhea outbreak caused by $C$. jejuni in the latest 2 decades in China.

\section{Conclusion}

This outbreak highlights that the danger of Campylobacter infection may lead to the occurrence of similar outbreaks in the future. Molecular analysis allowed for the detection of the outbreak strain with ST2274 and SMA001 for the first time, which could represent a marker to identify the source of infection if the outbreak investigation had been carried out in a timely manner. These data also suggest that authorities need to execute systematic surveillance and mandatory notification for Campylobacter, which is useful for the identification of the source of infection and proper control measures to protect public health.

\section{Acknowledgements}

We thank the staffs of Xicheng district CDC for collecting epidemiological information and sampling during the execution of this investigation. This work was supported by a grant from the National Key Program for Infectious Disease of China (2018ZX10714002-003-002).

\section{References}

1. Taylor EV, Herman KM, Ailes EC, Fitzgerald C, Yoder JS, Mahon BE, Tauxe RV (2013) Common source outbreaks of Campylobacter infection in the USA, 1997-2008. Epidemiol Infect 141: 987-996. 
2. Kaakoush NO, Castano-Rodriguez N, Mitchell HM, Man SM (2015) Global epidemiology of Campylobacter infection. Clin Microbiol Rev 28: 687-720.

3. Nohra A, Grinberg A, Midwinter AC, Marshall JC, CollinsEmerson JM, French NP (2016) Molecular epidemiology of Campylobacter coli strains isolated from different sources in New Zealand between 2005 and 2014. Appl Environ Microbiol 82: 4363-4370.

4. Silva J, Leite D, Fernandes M, Mena C, Gibbs PA, Teixeira P (2011) Campylobacter spp. as a foodborne pathogen: A review. Front Microbiol 2: 200.

5. Maertens de Noordhout C, Devleesschauwer B, Haagsma JA, Havelaar AH, Bertrand S, Vandenberg O, Quoilin S, Brandt PT, Speybroeck N (2017) Burden of salmonellosis, campylobacteriosis and listeriosis: a time series analysis, Belgium, 2012 to 2020. Euro Surveill 22.

6. Bresee JS, Marcus R, Venezia RA, Keene WE, Morse D, Thanassi M, Brunett P, Bulens S, Beard RS, Dauphin LA, Slutsker L, Bopp C, Eberhard M, Hall A, Vinje J, Monroe SS, Glass RI, Team USAGES (2012) The etiology of severe acute gastroenteritis among adults visiting emergency departments in the United States. J Infect Dis 205: 1374-1381.

7. Scallan E, Hoekstra RM, Angulo FJ, Tauxe RV, Widdowson MA, Roy SL, Jones JL, Griffin PM (2011) Foodborne illness acquired in the United States--major pathogens. Emerg Infect Dis 17: 7-15.

8. Revez J, Llarena AK, Schott T, Kuusi M, Hakkinen M, Kivisto R, Hanninen ML, Rossi M. (2014) Genome analysis of Campylobacter jejuni strains isolated from a waterborne outbreak. BMC Genomics 15: 768.

9. Eurosurveillance editorial team (2013) The European Union summary report on trends and sources of zoonoses, zoonotic agents and food-borne outbreaks in 2011 has been published. Euro Surveill 18: 20449.

10. Shen H, Zhang J, Li Y, Xie S, Jiang Y, Wu Y, Ye Y, Yang H, Mo H, Situ C, Hu Q (2016) The 12 gastrointestinal pathogens spectrum of acute infectious diarrhea in a sentinel hospital, Shenzhen, China. Front Microbiol 7: 1926.

11. Chang H, Zhang L, Ge Y, Cai J, Wang X, Huang Z, Guo J, Xu H, Gu Z, Chen H, Xu X, Zeng M (2017) A hospital-based casecontrol study of diarrhea in children in Shanghai. Pediatr Infect Dis J 36: 1057-1063.

12. Li Y, Zhang S, He M, Zhang Y, Fu Y, Liang H, Jing H, Li Y, Ma H, Zhang M (2018) Prevalence and molecular characterization of Campylobacter spp. isolated from patients with diarrhea in Shunyi, Beijing. Front Microbiol 9: 52.

13. Dingle KE, Colles FM, Wareing DR, Ure R, Fox AJ, Bolton FE, Bootsma HJ, Willems RJ, Urwin R, Maiden MC (2001) Multilocus sequence typing system for Campylobacter jejuni. J Clin Microbiol 39: 14-23.

14. Yu JH, Kim NY, Cho NG, Kim JH, Kang YA, Lee HG (2010) Epidemiology of Campylobacter jejuni outbreak in a middle school in Incheon, Korea. J Korean Med Sci 25: 1595-1600.

15. Man SM (2011) The clinical importance of emerging Campylobacter species. Nat Rev Gastroenterol Hepatol 8: 669685.

16. Bullman S, O'Leary J, Corcoran D, Sleator RD, Lucey B (2012) Molecular-based detection of non-culturable and emerging campylobacteria in patients presenting with gastroenteritis. Epidemiol Infect 140: 684-688.

17. Klein-Jobstl D, Sofka D, Iwersen M, Drillich M, Hilbert F (2016) Multilocus sequence typing and antimicrobial resistance of Campylobacter jejuni isolated from dairy calves in Austria. Front Microbiol 7: 72.

18. Zhang M, Liu X, Xu X, Gu Y, Tao X, Yang X, Yan G, Zhang J (2014) Molecular subtyping and antimicrobial susceptibilities of Campylobacter coli isolates from diarrheal patients and food-producing animals in China. Foodborne Pathog Dis 11: 610-619.

19. Zhang A, Song L, Liang H, Gu Y, Zhang C, Liu X, Zhang J, Zhang M (2016) Molecular subtyping and erythromycin resistance of Campylobacter in China. J Appl Microbiol 121: 287-293.

20. Burakoff A, Brown K, Knutsen J, Hopewell C, Rowe S, Bennett C, Cronquist A (2018) Outbreak of fluoroquinoloneresistant Campylobacter jejuni infections associated with raw milk consumption from a herdshare dairy - Colorado, 2016. MMWR Morb Mortal Wkly Rep 67: 146-148.

21. Zhang M, Gu Y, He L, Ran L, Xia S, Han X, Li H, Zhou H, Cui Z, Zhang J (2010) Molecular typing and antimicrobial susceptibility profiles of Campylobacter jejuni isolates from north China. J Med Microbiol 59: 1171-1177.

22. Li B, Ma L, Li Y, Jia H, Wei J, Shao D, Liu K, Shi Y, Qiu Y, Ma Z (2017) Antimicrobial resistance of Campylobacter species isolated from broilers in live bird markets in Shanghai, China. Foodborne Pathog Dis 14: 96-102.

23. Zhang T, Luo Q, Chen Y, Li T, Wen G, Zhang R, Luo L, Lu Q, Ai D, Wang H, Shao H (2016) Molecular epidemiology, virulence determinants and antimicrobial resistance of Campylobacter spreading in retail chicken meat in Central China. Gut Pathog 8: 48.

24. Ju CY, Zhang MJ, Ma YP, Lu JR, Yu MH, Chen H, Liu CY, Gu YX, Fu YY, Duan YX (2018) Genetic and antibiotic resistance characteristics of Campylobacter jejuni isolated from diarrheal patients, poultry and cattle in Shenzhen. Biomed Environ Sci 31: 579-585.

25. Strachan NJ, Rotariu O, Smith-Palmer A, Cowden J, Sheppard SK, O'Brien SJ, Maiden MC, Macrae M, Bessell PR, Matthews L, Reid SW, Innocent GT, Ogden ID, Forbes KJ (2013) Identifying the seasonal origins of human campylobacteriosis. Epidemiol Infect 141: 1267-1275.

26. Nielsen EM, Engberg J, Fussing V, Petersen L, Brogren CH, On SL (2000) Evaluation of phenotypic and genotypic methods for subtyping Campylobacter jejuni isolates from humans, poultry, and cattle. J Clin Microbiol 38: 3800-3810.

27. Wang J, Guo YC, Li N (2013) Prevalence and risk assessment of Campylobacter jejuni in chicken in China. Biomed Environ Sci 26: 243-248.

28. Zhang X, Tang M, Zhou Q, Zhang J, Yang X, Gao Y (2018) Prevalence and characteristics of Campylobacter throughout the slaughter process of different broiler batches. Front Microbiol 9: 2092.

29. Ma H, Su Y, Ma L, Ma L, Li P, Du X, Golz G, Wang S, Lu X (2017) Prevalence and characterization of Campylobacter jejuni isolated from retail chicken in Tianjin, China. J Food Prot 80: 1032-1040.

30. Boysen L, Rosenquist H, Larsson JT, Nielsen EM, Sorensen G, Nordentoft S, Hald T (2014) Source attribution of human campylobacteriosis in Denmark. Epidemiol Infect 142: 15991608.

31. Sheppard SK, Dallas JF, Strachan NJ, MacRae M, McCarthy ND, Wilson DJ, Gormley FJ, Falush D, Ogden ID, Maiden MC, Forbes KJ (2009) Campylobacter genotyping to determine the source of human infection. Clin Infect Dis 48: 1072-1078. 
32. Hanninen ML, Hakkinen M, Rautelin H (1999) Stability of related human and chicken Campylobacter jejuni genotypes after passage through chick intestine studied by pulsed-field gel electrophoresis. Appl Environ Microbiol 65: 2272-2275.

33. Scott AE, Timms AR, Connerton PL, Loc Carrillo C, Adzfa Radzum K, Connerton IF (2007) Genome dynamics of Campylobacter jejuni in response to bacteriophage predation. PLoS Pathog 3: e119.

34. Clark CG, Price L, Ahmed R, Woodward DL, Melito PL, Rodgers FG, Jamieson F, Ciebin B, Li A, Ellis A (2003) Characterization of waterborne outbreak-associated Campylobacter jejuni, Walkerton, Ontario. Emerg Infect Dis 9: 1232-1241.

\section{Corresponding author}

Quanyi Wang

Institute of Infectious and Endemic Diseases Control

Beijing Centre for Disease Prevention and Control, Beijing

Research Center for Preventive Medicine, No. 16, Hepingli Middle

Street, Beijing, 100013, China

Tel: +86-10-64407109

Fax: +86-10-64407113

E-mail: bjcdcxm@126.com

Conflict of interests: No conflict of interests is declared. 